\title{
Bioactive extracellular matrix fragments in lung health and disease
}

\author{
Amit Gaggar ${ }^{1,2,3}$ and Nathaniel Weathington ${ }^{4}$ \\ 'University of Alabama at Birmingham, Department of Medicine, Division of Pulmonary and Critical Care Medicine, ${ }^{2}$ Birmingham Veterans Affairs Medical Center, and ${ }^{3}$ Program in Protease and Matrix \\ Biology, Birmingham, Alabama, USA. ${ }^{4}$ University of Pittsburgh, Department of Medicine, Division of Pulmonary, Allergy, and Critical Care Medicine, Pittsburgh, Pennsylvania, USA.
}

\begin{abstract}
The extracellular matrix (ECM) is the noncellular component critical in the maintenance of organ structure and the regulation of tissue development, organ structure, and cellular signaling. The ECM is a dynamic entity that undergoes continuous degradation and resynthesis. In addition to compromising structure, degradation of the ECM can liberate bioactive fragments that cause cellular activation and chemotaxis of a variety of cells. These fragments are termed matrikines, and their cellular activities are sentinel in the development and progression of tissue injury seen in chronic lung disease. Here, we discuss the matrikines that are known to be active in lung biology and their roles in lung disease. We also consider the use of matrikines as disease markers and potential therapeutic targets in lung disease.
\end{abstract}

\section{Introduction}

The extracellular matrix (ECM) is the component in solid organs that serves as a scaffold for constituent cells and as a crucial regulator of cell-to-cell interactions that are important for cellular homeostasis and organ function. ECM is a versatile and dynamic compartment that actively synthesizes, degrades, and remodels its components (1). Throughout development and in adulthood, this plasticity can modulate ECM composition, tissue mechanical properties, and the behavior of associated cells, as well as provide damage response signals while maintaining organ function (2).

During lung development, the airways and alveoli form through multiple septation events, and the activity of both cellular ECM synthesis and proteases regulates the complex biology of branching morphogenesis $(3,4)$. ECM synthetic processes at the leading edge of developing alveoli are fine-tuned by concurrent expression of elastase (5), collagenases, the laminin-cleaving metalloprotease MMP14 (6), and hyaluronidase (7) during organ morphogenesis. Elastins are the most abundant ECM protein in lung, and are thought to be primarily synthesized on a stromal scaffold during this alveolar development without new synthesis over a mammal's lifetime $(8,9)$. Collagens represent almost $10 \%$ of the total protein mass of the adult mammalian lung, with maximal synthesis occurring during growth to adult size as well as continuous lower-level collagen synthesis ongoing through adult life $(10,11)$. Some lytic enzymes are also differentially expressed through life. In mature animals, the MMP9 collagenase is detectable in inflammatory and homeostatic cell types (12), while elastases seem to be induced mainly during inflammation or injury responses (9). Other ECM constituents like hyaluronan and laminin molecules undergo considerably more rapid turnover than the structural collagen and elastin

Conflict of interest: The authors have declared that no conflict of interest exists. Reference information: J Clin Invest. 2016;126(9):3176-3184. doi:10.1172/JCI83147. megaproteins, with ongoing synthesis and degradation that is accelerated in areas of tissue damage or inflammation $(13,14)$.

Dynamic remodeling of the ECM is required for development, wound healing, and normal organ responses; however, excessive or uncontrolled remodeling is a contributing factor in a variety of disease states (15). During remodeling, the ECM undergoes proteolytic processing that releases bioactive matrix fragments. Such bioactive matrix fragments have been appreciated since the $1970 \mathrm{~s}$ and were termed "matrikines" by Maquart and Monboisse (16, 17). These matrikines have been shown to promote cellular infiltration, progressive tissue damage, or wound healing. Thus, these signals represent an important effector mechanism of the ECM for cell signaling and trafficking of cells into target organs.

In the lung, the diversity of matrikine signals matches that of the parent matrix molecules from which they are derived, as structural proteins like collagen and elastin can invoke leukocyte activation and migration after their cleavage, and the degradation products of basement membrane constituents like laminin and the polysaccharide hyaluronan serve as signaling mediators to the epithelia. Inflammatory signals exerted by matrikines have been under study by multiple investigators for nearly 50 years, with clear indications that tissue damage recruits inflammatory cells to the site of injury. Based on these findings, we consider these matrikines to serve the same role as classical damage-associated molecular patterns (DAMPs). Thus, matrikines may be long-standing alarm signals in animal host defenses, as they represent a functional advance in intercellular communication during the evolution from single-celled to multicelled organisms. This Review will describe our current understanding of the specific mechanisms and phenotypes modulated by matrikines observed in the lung. Specific attention will be paid to detection of these fragments in human lung disease and potential therapeutic targeting to improve lung disease outcomes.

\section{Evolution and remodeling of the ECM}

The development and diversification of ECM is considered a critical feature characterizing evolution from single-celled to multicellular 
organisms, though structural carbohydrate-based ECM constituents (like hyaluronan) are present in life as primitive as bacteria. The metazoan ECM, including that of mammals, contains components present in species that appeared prior to choanoflagellates, suggesting that these conserved ECM constituents maintain integrity and signaling in tissues in all animals and predate the evolution of the adaptive immune system in animals. Some mammalian ECM constituents demonstrate increased diversification relative to the ancient metazoans, likely secondary to exonic shuffling (18).

The healthy ECM is maintained in a dynamic steady state that requires both active generation and regulated degradation of protein and carbohydrate components. As ECM has evolved, so have the lytic enzymes that degrade it. There is an increased appreciation for the diversity of proteases in the mammalian genome and, as a result, the varied biologic functions of these enzymes in maintenance of ECM. The protease enzymes are classified into 6 major structural groups: aspartic and glutamic proteases; serine, cysteine, and threonine proteases; and metalloproteases. There are multiple family members within each group, with some conserved elements across species and some proteases that are either expanded or inactivated over the course of evolution. Likewise, the carbohydrates chondroitin sulfate and hyaluronan exhibit similar ubiquity with much less diversity through the animal kingdom; these molecules date as far back as cephalochordata. Hyaluronic acid synthase and hyaluronidase enzymes modulate the deposition and resorption of the structural glycosaminoglycan hyaluronan, which constitutes the basement membrane (19). Considerable diversity is also present in gene expression patterns, and there is additional evolutionary complexity linked to activation and inhibition of matrix lytic enzymes among different species. Furthermore, there is both redundancy and promiscuity in protease-substrate targeting. Thus, predicting the impact of a given enzyme on ECM modulation across multiple genera or species represents a significant analytical challenge (20).

\section{Matrikines defined}

We use 3 criteria to define matrikines. (a) Matrikines are biomolecules derived from larger ECM macromolecules such as hyaluronan, collagen, or laminin. (b) They are generated through enzymatic or chemical cleavage (and possibly other modification) of the parent molecule. (c) They serve as a physiologic signal for cells or cellular receptors that are not activated by the full-sized parent matrix macromolecule.

\section{Collagen-derived matrikines}

Collagen represents the most abundant protein found in mammals and accounts for over $90 \%$ of the total protein mass in the ECM (21). Collagen fibers provide tensile strength to the ECM and maintain scaffolding for cell-to-cell communication. Twenty-eight different collagens containing 46 distinct polypeptide chains have been identified in vertebrates (22). Collagen polypeptides possess a primary structural Gly-Pro-X repeat (with X often being another proline) that is responsible for their characteristic right-handed helix secondary structure. The quaternary structure of collagen is a trimeric triple helix that contains $2 \alpha_{1}$ chains and $1 \alpha_{2}$ chain. Interstrand hydrogen bonding between proline and hydroxyproline buries the abundant glycine residues (at least one-third of the protein residues) within this trimeric collagen molecule, making them largely inaccessible in solution, and thus protected from enzymatic degradation. This triple-helix strand can then be further assembled into either nonfibrillar collagen (type IV) or fibrillar collagen (types I, II, III, and V), with each fibril containing many collagen molecules. Protection (23) of the core glycine residues from proteolysis is important to maintain the structural integrity of collagen (24), and many inherited disorders of ECM stability, including osteogenesis imperfecta, are related to amino acid substitution of collagen glycines $(25,26)$.

Collagen is synthesized and degraded in the lung constitutively and during inflammation and wound healing, resulting in the generation of multiple proteolytic fragments in the pulmonary interstitium that can diffuse to alveolar spaces, airways, and the bloodstream. Collagen types I and III are the predominant isoforms found in the lungs $(27,28)$, though types IV and V are also expressed (29) and are critical for lung homeostasis. Intact type IV and $\mathrm{V}$ collagens interact with integrins (30), matricellular proteins (31), and discoidin receptor (32) in normal physiology.

Early observations suggested that degraded collagen could induce cellular influx, but varied in their characterization of cell types. Fragmented collagen was shown to be chemotactic for rat neutrophils (or polymorphonuclear leukocytes [PMNs]) in vivo (33), while Postlethwaite and Kang demonstrated that collagenase-mediated degradation of type I collagen induced monocyte but not PMN chemotaxis (34). Still other studies suggested that other cell types, such as fibroblasts, can chemotax toward degraded type I, II, or III collagens (35). Specific collagen sequences and cellular factors regulating this activity were largely unexplored in these studies.

Proline-glycine-proline: a multifaceted matrikine. Early studies demonstrated that native collagen fragmentation could induce PMN chemotaxis both in vitro and in vivo $(36,37)$, and these studies further determined that pentameric peptides of synthetic collagen containing prolines, glycines, and hydroxyprolines could induce PMN chemotaxis and activation of the inflammatory transcriptional activator NF- $\mathrm{kB}$ (38). The tripeptide proline-glycine-proline (PGP) was first characterized as a matrikine by Pfister and colleagues during their investigation of persistent neutrophilic inflammation after ocular alkali injury, a condition characterized by significant ECM turnover. They discovered that both N-terminal acetylated PGP (N-ac-PGP) and N-terminal methylated PGP (N-me-PGP) peptides are present in this injury model (39) and that N-ac-PGP is chemotactic for neutrophils both in vitro (40) and in vivo (41), mimicking the neutrophilic influx observed in alkali-mediated eye injury. A peptide antagonist of PGP peptides, tetramerized arginine-threonine-arginine (RTR), markedly attenuated both neutrophil influx and corneal ulceration after alkali injury in rabbits (42). These results implicated the PGP tripeptide in neutrophilic inflammation from chemical injury and suggested that PGP may be present and active in other inflammatory diseases that are characterized by neutrophil influx.

In 2006, we determined that $\mathrm{N}$-ac-PGP induced PMN chemotaxis by ligating the chemokine receptors CXCR1 and CXCR2, the same receptors ligated by glutamic acid-leucine-arginine motif-containing (ELR+ ${ }^{+}$CXC chemokines such as IL-8 (43). The ability of PGP peptides to mimic ELR ${ }^{+}$CXC chemokines is due to structural homology between PGP and key sequences required for chemokine receptor binding. PGP peptides act as a partial agonist of CXCR1 and CXCR2, triggering PMN infiltration in vitro and 


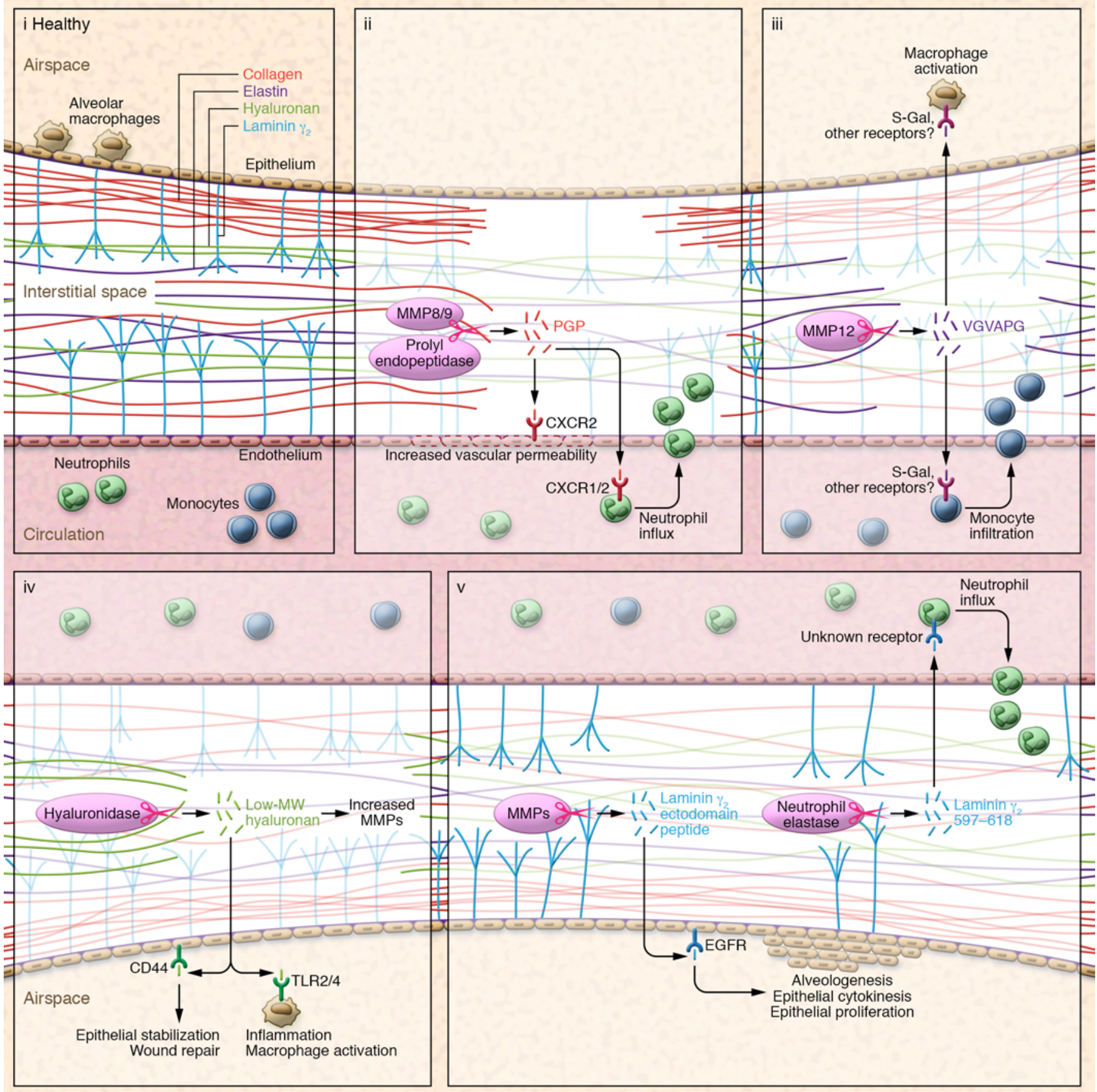

Figure 1. Matrikine signals in lung physiology. (i) ECM structural fibrous proteins collagen and elastin, the basement membrane integral protein laminin 332, and the glycosaminoglycan hyaluronan are all degraded by lytic enzymes to expose cryptic molecular fragments (matrikines), which activate signals on nearby cells to influence physiology. (ii) MMP9 and/or MMP8 cleave ECM collagen to form fragments that are further processed by prolyl endopeptidase to release PGP, which ligates CXCR1 and CXCR2 chemokine receptors to mediate neutrophil chemotaxis into the area of injury and increase vascular permeability. (iii) Elastin fragmentation, primarily mediated by MMP12, generates VGVAPG peptides, which are chemoattractant for monocytes and are proposed to act through the S-Gal receptor. (iv) HA within basement membranes is cleaved by hyaluronidase isoforms during tissue injury to release lowmolecular weight, tissue-active HA, which ligates CD44 and TLRs on epithelial cells and leukocytes to invoke appropriate inflammatory and wound healing responses to injury and prevent excessive tissue remodeling and fibrosis. (v) Laminin $332 \gamma$ subunit in basement membranes is cleaved by multiple MMPs with release of the $\gamma_{2}$ ectodomain peptide, an EGFR ligand that promotes epithelial proliferation to promote alveologenesis and tissue remodeling.

in vivo in the lungs. While PGP is suspected to be the smallest sequence capable of ligating CXCR1 and CXCR2 in vitro, other fragments and PGP-containing sequences have yet to be tested for their ability to signal through CXCRs or other leukocyte receptors.
PGP is liberated from collagen first by the action of the matrix metalloproteases MMP8 and MMP9, followed by the terminal action of the serine protease prolyl endopeptidase (44). Prolyl endopeptidase was previously considered a cytosolic housekeep- 
ing protein for structural and immune cells, but is now considered the rate-limiting enzyme for free PGP formation and is released from airway epithelia via an exosomal pathway during inflammation (45). PGP-induced signaling is considered a feed-forward inflammatory signal wherein CXCR1 and CXCR2 ligation triggers neutrophil influx, driving the release of collagenase MMP9 from neutrophil tertiary granules, which cleaves more collagen and results in the release of additional PGP and subsequent neutrophil influx (46) (Figure 1).

PGP in the interstitium and airway is normally cleared by further enzymatic degradation, and PGP-mediated inflammation is self-limited. Using a murine model of influenza virus infection, Snelgrove and colleagues discovered that PGP is cleaved and inactivated by the leukotriene A4 hydrolase (LTA4H) (47). LTA4H is better known for hydrolyzing leukotriene A4 into leukotriene B4 (LTB4), a potent lipid chemoattractant and broad activator of immune cells, including PMNs, mast cells, NK cells, B cells, T cells, and macrophages (48). LTA4H also exhibits aminopeptidase activity that cleaves PGP, but not acetylated PGP (Ac-PGP), and this protease action is distinct from the known lipid hydrolytic actions. Thus, LTA4H is a dual proinflammatory and anti-inflammatory enzyme. Blocking LTA4H aminopeptidase activity increases PGP levels and neutrophil influx in mice treated with influenza virus.

Long-term Ac-PGP administration is sufficient to cause lung tissue destruction and emphysema in mice, a condition that mimics chronic obstructive pulmonary disease (COPD) in humans. Biologically relevant quantities of Ac-PGP are generated in vivo by collagen destruction in acute (murine LPS inhalation) and chronic inflammatory injury, such as occurs in human $\operatorname{COPD}(43,49)$. The RTR antagonist (described above) abrogated PMN infiltration into mouse lungs in response to Ac-PGP exposure, and also prevented LPS- or cigarette smoke-induced neutrophilia, weakly suppressed in vitro IL-8-dependent neutrophil chemotaxis, and rescued emphysema after administration of either Ac-PGP or LPS $(49,50)$. As was observed in $\operatorname{COPD}(51,52), \mathrm{PGP}$ is upregulated in many other human lung diseases, including cystic fibrosis (CF) exacerbation (44), bronchiolitis obliterans syndrome (53), and acute respiratory distress syndrome (ARDS) $(47,54)$. Notably, cigarette smoke (CS) influences PGP activity via LTA4H. The CS component acrolein potently inhibits LTA4H aminopeptidase activity without affecting LTA4H hydrolase activity (55), while CS enhances the conversion of PGP to Ac-PGP (55). Thus, CS promotes PGP peptide accumulation by impairing LTA4H peptidase activity and chemically stabilizing PGP via acetylation, all the while permitting LTB4 biosynthesis to augment inflammation in lung disease.

In lung and other tissues, PGP peptides can act on any cells that express the appropriate CXCR. Endothelial CXCR2 signaling increases vascular permeability via PGP peptides in vitro and in vivo (54). Ac-PGP causes migration and differentiation of cartilage endplate stem cells in degenerated intravertebral discs (56). The effect of PGP signaling on other cell types such as fibroblasts and epithelial cells is currently under study.

\section{Elastin-derived matrikines}

Elastin is a polymer of tropoelastin that consists of lysine- and alanine-rich domains along with hydrophobic domains that are rich in glycine, valine, and proline residues, which occur in repeats. Elastin plays a critical role in lung physiology, functioning to maintain lung elastic recoil and thus ventilation. However, as lung structural development is completed early in life, elastin expression is downregulated with age, highlighting the need for the preservation of primary elastin to maintain optimal lung function. Indeed, the principal clinical findings of emphysematous COPD include hypoventilation and loss of lung elastic recoil, which are related to loss of elastin.

In addition to compromising structural integrity, proteolytic degradation of elastin enhances ongoing pulmonary injury by liberating matrikines. Early studies by both Senior and colleagues and Hunninghake and colleagues demonstrated that elastin fragments under $50 \mathrm{kDa}$ were capable of potently inducing monocyte chemotaxis $(57,58)$. In 1984, Senior and colleagues examined the elastin repeating hexamer VGVAPG and found the peptide to be chemotactic to both monocytes and fibroblasts (59). Further studies demonstrated that this peptide upregulates various MMPs in endothelial cells, fibroblasts, and tumor cells (60-63). The conformation of VGVAPG is critical to its biologic function (64), and the S-Gal receptor (also known as elastin-binding protein $[\mathrm{EBP}])$ is thought to be the primary elastin receptor for this fragment (65) (Figure 1).

A variety of proteases degrade elastin, and their tight regulation is critical for the maintenance of pulmonary health. Elastin-derived peptides are likely elicited by the elastases macrophage metalloelastase (MMP12) and neutrophil elastase $(66,67)$. Both the lungs of individuals deficient in $\alpha_{1}$-antitrypsin, which blocks the activity of neutrophil elastase, and murine lungs instilled with elastinolytic enzymes $(68,69)$ exhibit severely decreased lung elastance and augmented tissue degradation, providing the basis for a protease/antiprotease imbalance as a driver of lung disease $(70,71)$. MMP12 expression is highly upregulated in human smokers compared with nonsmokers (72). Hautamaki and colleagues described a critical role of MMP12 in emphysema, demonstrating that $M m p 12^{-/-}$mice are resistant to CS-induced emphysema because of decreased tissue destruction, loss of elastin matrikine signals, or both (73). These results were extended in a study of short-term CS exposure in mice, where inhibition of elastin fragments by neutralizing antibodies attenuated lung monocyte infiltration. In the same study, the neutralizing antibody attenuated macrophage infiltration and experimental emphysema after instillation of porcine pancreatic elastase (74).

MMP12 activity levels are also important in human obstructive lung disease. A large multicohort genotyping study identified an SNP within the promoter of the MMP12 gene that decreased transcription of the protease (75). Longitudinal follow-up data from over 8,000 individuals revealed that individuals homozygous or heterozygous for this SNP were significantly protected from acquiring COPD, irrespective of smoking status. Though this study did not directly measure elastin fragmentation or matrikine production, it is highly likely that elastin cleavage by specific proteases (especially MMP12) in response to insult directs feed-forward inflammation to drive lung disease in mice and humans. Indeed the MMP12 SNP and $\alpha_{1}$-antitrypsin mutations are the best-described genetic predictors for COPD risk stratification in people, and these findings further validate the hypothesis that imbalances in protease/antiprotease activities contribute significantly to obstructive lung disease pathogenesis. 
Table 1. Lung matrikines and associated biologic activity

\begin{tabular}{|c|c|c|c|c|c|}
\hline ECM constituent & Lytic enzyme & Matrikine & Receptor & Action & Select references \\
\hline \multirow[t]{2}{*}{ Collagen } & MMP8, -9 & $\mathrm{~N}$-ac-PGP, PGP & CXCR1 and CXCR2 & PMN influx & $39-47,54,101$ \\
\hline & Prolyl endopeptidase & & CXCR2 & Vascular permeability & \\
\hline Elastin & MMP12, neutrophil elastase & VGVAPG, & S-Gal, others? & Monocyte influx & $57-59,64-69$ \\
\hline Laminin 332 (aka laminin 5) & $\begin{array}{c}\text { MMP3, }-12,-14,-20, \text { neutrophil } \\
\text { elastase }\end{array}$ & $\gamma_{2}$ ectodomain & EGFR & $\begin{array}{l}\text { Epithelial proliferation, wound } \\
\text { repair, alveologenesis }\end{array}$ & $93-98$ \\
\hline
\end{tabular}

\section{Hyaluronan-derived matrikines}

Hyaluronan (HA) is an anionic glycosaminoglycan found throughout the ECM and comprises a large constituent of the basement membrane in solid organs. It is usually synthesized as a very large repeating polysaccharide composed of $\mathrm{N}$-acetyl-D-glycosamine and D-glucuronic acid that is widely present in soft tissues. As a functional component of ECM, HA can deform in any direction, act as a lubricant in joints, absorb water, and chemically scavenge ROS $(76,77)$. There are 3 HA synthase isoforms (HAS1-3) and 6 human hyaluronidase enzymes that degrade HA by hydrolysis of saccharide linkages (19). Exogenous hyaluronidases produced by bacteria or concentrated in snake and scorpion venom are pathologic factors that mediate tissue invasion and necrosis (13).

The molecular weight of HA is a primary determinant of its function as a matrikine. Like collagen and elastin, the common high-molecular weight ( $>500 \mathrm{kDa}) \mathrm{HA}$ is important for its tissue structural characteristics. The low-molecular weight $(<100 \mathrm{kDa})$ HA isoforms can ligate receptors to mediate cellular signaling activity. Thus, the availability of tissue-active HA is regulated by the degree of HA hydrolysis or synthesis. Among HA's effects, the induction of proteases or other matrix lytic enzymes is particularly relevant to its pathologic role. Low-molecular weight HA induces transcriptional upregulation and release of active forms of MMP12 (which cleaves elastin) and MMP9 (which cleaves collagen), each of which can facilitate further matrikine generation (78-80). HA activity impacts multiple aspects of cell migration, invasion, angiogenesis, and cellular differentiation; these activities of HA in lung physiology involve ligation of specific cellular receptors $(76,81,82)$.

CD44 is a ubiquitously expressed type I transmembrane glycoprotein that was the first characterized HA receptor (83). HA/ CD44 ligation is required for the resolution of acute pulmonary inflammation in animal models, with CD44-deficient mice developing severe postinflammatory fibrosis after bleomycin treatment (84). However, the diverse actions of HA are not fully explained by CD44 signaling, as some cells lacking CD44 (macrophages, epithelia, tumor cells, and others) respond to HA (80, 85). Lowmolecular weight forms of HA induce cytokine production and a proinflammatory phenotype, whereas larger HA fragments maintain an epithelial protective phenotype via ligation of TLR 4 and TLR2 (53), similar to the homeostatic epithelial TLR signaling in the gut, which maintains both appropriate inflammatory signaling and epithelial integrity (86). Based on these studies, there is an emerging paradigm in which multifaceted HA matrikine sig- naling through TLR ligation in the comparatively sterile microenvironment of the lung maintains appropriate low-level molecular pattern recognition signals on various cell types to mediate proper tissue responses and optimize repair, thereby preventing exuberant remodeling and fibrosis (Figure 1).

\section{Laminin-derived matrikines}

Laminins are large-molecular weight heterotrimeric glycoproteins that are designated with 3 number codes that correspond to their constituent $\alpha, \beta$, and $\gamma$ chains (e.g., laminin 111 or 543 ) (87). These proteins are found in large quantities in tissue basement membranes (88), where the C-terminus of the laminin $\alpha$ subunit interacts with basolateral cellular plasma membranes and the $\mathrm{N}$-termini of all 3 subunits assemble through coiled-coil domains and project into the basement membrane to interact with other ECM components such as collagen or HA. These glycoproteins transduce both biochemical and mechanical signals between ECM and cells to influence cell survival, differentiation, and migration (89).

Laminins are degraded by serine protease and metalloprotease family members $(90,91)$. Laminin 332 (also known as laminin 5 ) is a component of multiple basement membranes and is implicated in hemidesmosome formation (92). The cleavage of the $\gamma_{2}$ chain of laminin 332 by multiple MMPs (MMP3, -12, -13, -14, and -20) creates biologically active peptides that bind and ligate EGFR to mediate mitogenic epithelial activation (93) and cell migration (94-96). MMP14-dependent $\gamma_{2}$ cleavage in lung generates fragments with EGF mimetic activity that induces regenerative alveologenesis (97). Apart from metalloprotease activity, the serine protease neutrophil elastase has been shown to cleave all 3 chains of laminin 332, resulting in global neutrophil chemotaxis (98). More specifically, the neutrophil elastase-mediated cleavage of the $\gamma_{2}$ chain releases the $\gamma_{2}$ peptide 597-618. This peptide neighbors the MMP2 cleavage site and is chemotactic for PMNs in vitro, though the specific receptor for this peptide remains uncharacterized (Figure 1).

\section{Matrikines in health and disease}

Highly evolved ECM components in complex multicellular organisms provide unique biophysical properties to tissues with optimized organ-specific functions. In the lung, the ECM provides critical superstructure for robust gas exchange in an organ that is large, flexible, exposed to the outside environment, and perfused with the entire cardiac output. The roles of ECM in cell-to-cell 
communication, immune responses, tissue repair, and regeneration have coevolved within the organ, along with other tissue-specific, regulated biologic mediators such as cytokines.

Because the ECM functions as a scaffold for organ architectural stability and development, the interactions of various cell types with the molecules that make up the ECM are highly conserved and carefully orchestrated. Therefore, it logically follows that matrikines that result from abnormal cleavage of ECM molecules can initiate cell behaviors different from those that are triggered by interactions with intact ECM. The fact that a majority of these cellular responses occur after injury or infection and largely involve inflammatory activation and cell proliferation indicates that matrikines serve as primordial DAMPs. These cryptic patterns may persist in ECM as vestiges of ancient innate immune responses and can function as both sensor (through cleavage) and effector (through receptor binding) molecules for tissue-level stresses. To test this hypothesis, future studies could use modern analytical tools like mass spectrometry to search for matrikine signals in ancient organisms (e.g., invertebrates, early vertebrates, or early mammals) to determine whether a matrikine signal is present after injury or noxious exposure in lung or other tissues.

Matrikine peptides or polysaccharides are generally less potent than the cognate ligands for the receptors known to trigger immune or epithelial cell activation; for example, in PMN assays IL-8 binds and activates CXCRs at nanomolar concentrations (99), while micromolar concentrations of PGP are needed to activate cells (39). Nonetheless, the quantity and variety of these bioactive lytic products in the injured lung are sufficient for robust responses. Matrikine-induced DAMP responses in the acute setting appear to be highly tunable, as they are controlled by tightly regulated cellular and enzymatic activities that mediate the liberation of these bioactive fragments as well as their neutralization (100).

One compelling example of inflammatory matrikine activity was recently described during pulmonary Haemophilus influenzae infection, which generates PGP within hours and elastin fragments within days of infection in LTA4H-deficient mice. Each matrikine increased airway inflammation, and silencing of PGP prevented the later generation of elastin fragments. The interplay of these bioactive fragments in lung disease appears to be both temporally and spatially hierarchical (101). Future studies on matrikines in lung disease should correlate different stages of disease (i.e., clinical stability versus exacerbation) with measurements of matrikines, immune cells, and other cytokines in order to understand how matrikines influence known markers of disease progression.

While matrikine biology has largely been based on in vitro and murine studies, there is an emerging clinical literature exploring these messengers in human lung disorders. Mass spectrometry has been used to measure PGP peptides in clinical samples from patients with COPD $(43,51,52)$, CF, bronchiolitis obliterans syndrome, and ARDS $(44,53,54)$, with strong positive correlations seen between PGP levels and disease activity. PGP is now being measured as a dynamic biochemical readout in clinical trials involving patients with stable COPD and those suffering from CF exacerbation $(52,102)$. Similarly, other investigations using mass spectrometry show evidence of elastin fragmentation in samples from patients with COPD (103).
Collectively, these studies demonstrate the utility of mass spectrometry for the identification and quantification of the unique matrikines active in human disease.

Even with access to sophisticated mass spectrometry methods, ECM turnover in lung disease is difficult to specifically determine, making the impact of matrikine signaling challenging to study in humans with chronic lung disease. ECM components undergo synthesis $(104,105)$ concurrent with ECM breakdown, and each process is influenced by various factors such as increased mechanical stress (106) and tissue aging (107). Thus, it is challenging to identify matrikines that can exert a specific phenotype in diseases where the ECM undergoes continuous remodeling, as is seen in interstitial lung diseases including idiopathic pulmonary fibrosis, ARDS, and COPD. To our knowledge, the largest characterization of ECM turnover in lung disease was in the longitudinal monitoring of collagen protein turnover by neoepitope analysis in the PROFILE cohort of pulmonary fibrosis patients (108). This study used ELISA to detect and characterize collagen breakdown products and showed that patients with increased collagen breakdown products have worse outcomes from lung fibrosis in idiopathic pulmonary fibrosis. It has not yet been determined whether collagen fragments detected in these patients exert matrikine activity to propagate smoldering inflammation along with continuous remodeling, but the increased presence of collagen fragments is highly suggestive of a pathologic role. Further studies to characterize the biologic impact of these fragments on key readouts of leukocyte and fibroblast biology are warranted, and such studies may identify new therapeutic approaches for the treatment of fibrotic lung disorders.

Therapeutic targeting of matrikines. Modulation of matrikine levels could potentially influence outcomes in a variety of disease states. Such modulation could be achieved by targeting of the enzymes that generate or perpetuate a matrikine (MMP2, -8, -9, -12, -14 , prolyl endopeptidase, or hyaluronidase), targeting of the matrikine itself, or targeting of the putative receptors for the matrikines (Table 1). Indeed, a preclinical study of the dual MMP9/MMP12 inhibitor AZ11557272 shows protection against CS-induced lung damage in guinea pigs (109), reducing airway neutrophil and macrophage numbers and emphysema. By preventing both collagen and elastase degradation, such a compound would greatly reduce matrikine signaling, though measurements of PGP peptides or VGVAPG were not performed as part of the study. Direct targeting of both PGP peptides and elastin fragments in preclinical models of airway disease has suggested important roles for these peptides in disease pathology $(49,74,110)$. Interestingly, prior development of LTA4H inhibitors to attenuate LTB4 production did not consider the ability of this enzyme to inactivate PGP. New compounds that selectively inhibit the LTA4H hydrolase activity but leave the PGP-degrading aminopeptidase activity intact are being sought (111). To our knowledge, the only current direct matrikine inhibitor is the tetramerized peptide RTR, which has been shown to bind PGP and attenuate CS-induced lung injury in mice (49). RTR also weakly inhibits IL-8-induced PMN chemotaxis and attenuates endothelial permeability in human ARDS specimens (19), validating PGP and other CXCLs as therapeutic targets. Other potentially attractive matrikine targets include VGVAPG or the bioactive laminin 332 fragment, which, to our knowledge, are currently not being actively targeted within therapeutic platforms. 
Matrikines outside the lung. Though we focus on matrikines active in the lung, matrikine activity has been observed in other organs. In the skin, cleavage of collagen XVII by neutrophil elastase leads to a small PMN chemotactic fragment that contributes to the progression of experimental bullous pemphigoid in mice (112). Other studies have identified increased collagen fragmentation that can augment oxidative stress in skin fibroblasts, suggesting the presence of a matrikine-driven feed-forward loop for fibroblast activation (113). In vascular tissue, the cleavage of ECM laminins and integrins may expose cryptic Arg-Gly-Asp (RGD) motifs to recruit and activate inflammatory cells that can alter tissue reorganization during ECM resynthesis (114). As has been observed in the lung, ECM fragments of fibronectin, vitronectin, fibrinogen, and collagen can induce phagocytic functions and bactericidal activities of immune cells (115), highlighting DAMP functions of the matrikine-based immune response.

\section{Future directions}

As our tool set to dissect the complexities of matrikine signaling and regulation expands, it is likely that novel matrikine pathways with unique biologic activity will be identified. In considering ECM degradation as an injury response to reveal matrikine DAMPs, we can speculate that these signals may represent an arm of the immune system that predates and is partially precedent for the evolution of the molecular pattern receptors and chemokine systems found in modern vertebrates. Antagonizing this inflammatory pathway for therapy is an attractive notion; however, the matrikines that are active in pulmonary diseases also possess protective functions in promoting lung homeostasis that must be taken into consideration in designing therapeutic strategies to target these molecules.

\section{Acknowledgments}

We thank J.E. Blalock for his thoughtful reading of this manuscript. These studies were funded in part by grants from the NIH (HL102371 to A. Gaggar; HL126135 to N. Weathington), the Veterans Administration (1 I01 BX001756 to A. Gaggar), the American Lung Association (to N. Weathington), and the Ismail Moustapha Scholar Fund (to A. Gaggar).

Address correspondence to: Nathaniel Weathington, 3459 Fifth Avenue, MUH NW 628, Pittsburgh, Pennsylvania 15213, USA. Phone: 412.624.8669; E-mail: weathingtonnm@upmc.edu. Or to: Amit Gaggar, 1900 University Avenue THT 422, Birmingham, Alabama 35294, USA. Phone:205.934.6439; E-mail: agaggar@uabmc.edu.
1. Hynes RO. The extracellular matrix: not just pretty fibrils. Science. 2009;326(5957):1216-1219.

2. Freedman BR, et al. The (dys)functional extracellular matrix. Biochim Biophys Acta. 2015; 1853(11 pt B):3153-3164.

3. Daley WP, Peters SB, Larsen M. Extracellular matrix dynamics in development and regenerative medicine. JCell Sci. 2008;121(pt 3):255-264.

4. Bonnans C, Chou J, Werb Z. Remodelling the extracellular matrix in development and disease. Nat Rev Mol Cell Biol. 2014;15(12):786-801.

5. Liu S, Young SM, Varisco BM. Dynamic expression of chymotrypsin-like elastase 1 over the course of murine lung development. Am JPhysiol Lung Cell Mol Physiol. 2014;306(12):L1104-L1116.

6. Kheradmand F, Rishi K, Werb Z. Signaling through the EGF receptor controls lung morphogenesis in part by regulating MT1-MMP-mediated activation of gelatinase A/MMP2. JCell Sci. 2002;115(pt 4):839-848.

7. Menzel EJ, Farr C. Hyaluronidase and its substrate hyaluronan: biochemistry, biological activities and therapeutic uses. Cancer Lett. 1998;131(1):3-11.

8. Shapiro SD, Endicott SK, Province MA, Pierce JA, Campbell EJ. Marked longevity of human lung parenchymal elastic fibers deduced from prevalence of D-aspartate and nuclear weapons-related radiocarbon. J Clin Invest. 1991;87(5):1828-1834.

9. Kielty CM, Sherratt MJ, Shuttleworth CA. Elastic fibres. J Cell Sci. 2002;115(pt 14):2817-2828.

10. Bradley KH, McConnell SD, Crystal RG. Lung collagen composition and synthesis. Characterization and changes with age. J Biol Chem. 1974;249(9):2674-2683.

11. Bradley K, McConnell-Breul S, Crystal RG. Collagen in the human lung. Quantitation of rates of synthesis and partial characterization of composition. J Clin Invest. 1975;55(3):543-550.

12. Atkinson JJ, Senior RM. Matrix metalloprotein- ase-9 in lung remodeling. Am J Respir Cell Mol Biol. 2003;28(1):12-24.

13. Girish KS, Kemparaju K. The magic glue hyaluronan and its eraser hyaluronidase: a biological overview. Life Sci. 2007;80(21):1921-1943.

14. Hallmann R, Horn N, Selg M, Wendler O, Pausch F, Sorokin LM. Expression and function of laminins in the embryonic and mature vasculature. Physiol Rev. 2005;85(3):979-1000.

15. Chapman HA. Disorders of lung matrix remodeling. J Clin Invest. 2004;113(2):148-157.

16. Maquart FX, Pasco S, Ramont L, Hornebeck W, Monboisse JC. An introduction to matrikines: extracellular matrix-derived peptides which regulate cell activity. Implication in tumor invasion. Crit Rev Oncol Hematol. 2004;49(3):199-202.

17. Maquart FX, Simeon A, Pasco S, Monboisse JC. [Regulation of cell activity by the extracellular matrix: the concept of matrikines]. J Soc Biol. 1999;193(4-5):423-428.

18. Hynes RO. The evolution of metazoan extracellular matrix. J Cell Biol. 2012;196(6):671-679.

19. Csoka AB, Stern R. Hypotheses on the evolution of hyaluronan: a highly ironic acid. Glycobiology. 2013;23(4):398-411.

20. Folgueras AR, et al. Collagenase-2 deficiency or inhibition impairs experimental autoimmune encephalomyelitis in mice. J Biol Chem. 2008;283(14):9465-9474.

21. Kadler KE, Hill A, Canty-Laird EG. Collagen fibrillogenesis: fibronectin, integrins, and minor collagens as organizers and nucleators. Curr Opin Cell Biol. 2008;20(5):495-501.

22. Montes GS, Junqueira LC. Biology of collagen. Rev Can Biol Exp. 1982;41(2):143-156.

23. Exposito JY, Valcourt U, Cluzel C, Lethias C. The fibrillar collagen family. Int J Mol Sci. 2010;11(2):407-426.

24. Shoulders MD, Raines RT. Collagen structure and stability. Annu Rev Biochem. 2009;78:929-958.
25. Myllyharju J, Kivirikko KI. Collagens and collagen-related diseases. Ann Med. 2001;33(1):7-21.

26. Beck K, Chan VC, Shenoy N, Kirkpatrick A, Ramshaw JA, Brodsky B. Destabilization of osteogenesis imperfecta collagen-like model peptides correlates with the identity of the residue replacing glycine. Proc Natl Acad Sci US A. 2000;97(8):4273-4278.

27. McLees BD, Schleiter G, Pinnell SR. Isolation of type III collagen from human adult parenchymal lung tissue. Biochemistry. 1977;16(2):185-190.

28. Davidson JM. Biochemistry and turnover of lung interstitium. Eur Respir J. 1990;3(9):1048-1063.

29. Konomi H, Hayashi T, Nakayasu K, Arima M. Localization of type $\mathrm{V}$ collagen and type IV collagen in human cornea, lung, and skin. Immunohistochemical evidence by anti-collagen antibodies characterized by immunoelectroblotting. Am J Pathol. 1984;116(3):417-426.

30. Jokinen J, et al. Integrin-mediated cell adhesion to type I collagen fibrils. J Biol Chem. 2004;279(30):31956-31963.

31. Sidhu SS, et al. Roles of epithelial cell-derived periostin in TGF-beta activation, collagen production, and collagen gel elasticity in asthma. Proc Natl Acad Sci U S A. 2010;107(32):14170-14175

32. Xu H, Raynal N, Stathopoulos S, Myllyharju J, Farndale RW, Leitinger B. Collagen binding specificity of the discoidin domain receptors: binding sites on collagens II and III and molecular determinants for collagen IV recognition by DDR1. Matrix Biol. 2011;30(1):16-26.

33. Chang C, Houck JC. Demonstration of the chemotactic properties of collagen. Proc Soc Exp Biol Med.1970;134(1):22-26.

34. Postlethwaite AE, Kang AH. Collagen-and collagen peptide-induced chemotaxis of human blood monocytes. JExp Med. 1976;143(6):1299-1307.

35. Postlethwaite AE, Seyer JM, Kang AH. Chemotactic attraction of human fibroblasts to type I, II, 
and III collagens and collagen-derived peptides. Proc Natl Acad Sci U S A. 1978;75(2):871-875.

36. Riley DJ, Kerr JS, Guss HN, Curran SF, Laskin DL, Berg RA. Intratracheal instillation of collagen peptides induces a neutrophil influx in rat lungs. Trans Assoc Am Physicians. 1984;97:290-295.

37. Laskin DL, Kimura T, Sakakibara S, Riley DJ, Berg RA. Chemotactic activity of collagen-like polypeptides for human peripheral blood neutrophils. JLeukoc Biol. 1986;39(3):255-266.

38. Weinberger B, et al. Mechanisms mediating the biologic activity of synthetic proline, glycine, and hydroxyproline polypeptides in human neutrophils. Mediators Inflamm. 2005;2005(1):31-38.

39. Pfister RR, Haddox JL, Sommers CI, Lam KW. Identification and synthesis of chemotactic tripeptides from alkali-degraded whole cornea. A study of N-acetyl-proline-glycine-proline and $\mathrm{N}$-methyl-proline-glycine-proline. Invest $\mathrm{Oph}$ thalmol Vis Sci. 1995;36(7):1306-1316.

40. Pfister RR, Haddox JL. A neutrophil chemoattractant is released from cellular and extracellular components of the alkali-degraded cornea and blood. Invest Ophthalmol Vis Sci. 1996;37(1):230-237.

41. Pfister RR, Haddox JL, Sommers CI. Injection of chemoattractants into normal cornea: a model of inflammation after alkali injury. Invest Ophthalmol Vis Sci. 1998;39(9):1744-1750.

42. Haddox JL, Pfister RR, Sommers CI, Blalock JE, Villain M. Inhibitory effect of a complementary peptide on ulceration in the alkaliinjured rabbit cornea. Invest Ophthalmol Vis Sci. 2001;42(12):2769-2775.

43. Weathington NM, et al. A novel peptide CXCR ligand derived from extracellular matrix degradation during airway inflammation. Nat Med. 2006;12(3):317-323.

44. Gaggar A, et al. A novel proteolytic cascade generates an extracellular matrix-derived chemoattractant in chronic neutrophilic inflammation. J Immunol. 2008;180(8):5662-5669.

45. Szul T, et al. Toll-like receptor 4 engagement mediates prolyl endopeptidase release from airway epithelia via exosomes. Am J Respir Cell Mol Biol. 2016;54(3):359-369.

46. Xu X, et al. A self-propagating matrix metalloprotease-9 (MMP-9) dependent cycle of chronic neutrophilic inflammation. PLoS One. 2011;6(1):e15781.

47. Snelgrove RJ, et al. A critical role for LTA4H in limiting chronic pulmonary neutrophilic inflammation. Science. 2010;330(6000):90-94.

48. Hedi H, Norbert G. 5-Lipoxygenase pathway, dendritic cells, and adaptive immunity.J Biomed Biotechnol. 2004;2004(2):99-105.

49. van Houwelingen AH, Weathington NM, Verweij V, Blalock JE, Nijkamp FP, Folkerts G. Induction of lung emphysema is prevented by L-arginine-threonine-arginine. FASEB J. 2008;22(9):3403-3408.

50. Braber S, et al. Cigarette smoke-induced lung emphysema in mice is associated with prolyl endopeptidase, an enzyme involved in collagen breakdown. Am J Physiol Lung Cell Mol Physiol. 2011;300(2):L255-L265.

51. O’Reilly PJ, Jackson PL, Wells JM, Dransfield MT, Scanlon PD, Blalock JE. Sputum PGP is reduced by azithromycin treatment in patients with COPD and correlates with exacerbations. BMJ
Open. 2013;3(12):e004140.

52. Wells JM, et al. A randomized, placebo-controlled trial of roflumilast. Effect on proline-glycine-proline and neutrophilic inflammation in chronic obstructive pulmonary disease. Am J Respir Crit Care Med. 2015;192(8):934-942.

53. Hardison MT, et al. The presence of a matrixderived neutrophil chemoattractant in bronchiolitis obliterans syndrome after lung transplantation. JImmunol. 2009;182(7):4423-4431.

54 . Hahn CS, et al. The matrikine N- $\alpha$-PGP couples extracellular matrix fragmentation to endothelial permeability. Sci Adv. 2015;1(3):e1500175.

55. Noerager BD, et al. A potential role for acrolein in neutrophil-mediated chronic inflammation. Inflammation. 2015;38(6):2279-2287.

56. Feng C, Zhang Y, Yang M, Huang B, Zhou Y. Collagen-derived $\mathrm{N}$-acetylated proline-glycine-proline in intervertebral discs modulates CXCR1/2 expression and activation in cartilage endplate stem cells to induce migration and differentiation toward a pro-inflammatory phenotype. Stem Cells. 2015;33(12):3558-3568.

57. Senior RM, Griffin GL, Mecham RP. Chemotactic activity of elastin-derived peptides. JClin Invest. 1980;66(4):859-862.

58. Hunninghake GW, Davidson JM, Rennard S, Szapiel S, Gadek JE, Crystal RG. Elastin fragments attract macrophage precursors to diseased sites in pulmonary emphysema. Science. 1981;212(4497):925-927.

59. Senior RM, Griffin GL, Mecham RP, Wrenn DS, Prasad KU, Urry DW. Val-Gly-Val-Ala-Pro-Gly, a repeating peptide in elastin, is chemotactic for fibroblasts and monocytes. J Cell Biol. 1984;99(3):870-874.

60. Fahem A, et al. Elastokine-mediated up-regulation of MT1-MMP is triggered by nitric oxide in endothelial cells. Int J Biochem Cell Biol. 2008;40(8):1581-1596.

61. Brassart B, et al. Conformational dependence of collagenase (matrix metalloproteinase-1) up-regulation by elastin peptides in cultured fibroblasts. JBiol Chem. 2001;276(7):5222-5227.

62. Brassart B, Randoux A, Hornebeck W, Emonard H. Regulation of matrix metalloproteinase-2 (gelatinase A, MMP-2), membrane-type matrix metalloproteinase-1 (MT1-MMP) and tissue inhibitor of metalloproteinases-2 (TIMP-2) expression by elastin-derived peptides in human HT-1080 fibrosarcoma cell line. Clin Exp Metastasis. 1998;16(6):489-500.

63. Toupance S, et al. Elastin-derived peptides increase invasive capacities of lung cancer cells by post-transcriptional regulation of MMP- 2 and uPA. Clin Exp Metastasis. 2012;29(5):511-522.

64. Floquet N, Héry-Huynh S, Dauchez M, Derreumaux P, Tamburro AM, Alix AJ. Structural characterization of VGVAPG, an elastin-derived peptide. Biopolymers. 2004;76(3):266-280.

65. Mecham RP, Hinek A, Entwistle R, Wrenn DS, Griffin GL, Senior RM. Elastin binds to a multifunctional 67-kilodalton peripheral membrane protein. Biochemistry. 1989;28(9):3716-3722.

66. Bisaccia F, Morelli MA, De Biasi M, Traniello S, Spisani S, Tamburro AM. Migration of monocytes in the presence of elastolytic fragments of elastin and in synthetic derivates. Struc- ture-activity relationships. Int JPept Protein Res. 1994;44(4):332-341.

67. Taddese S, Weiss AS, Jahreis G, Neubert RH, Schmelzer CE. In vitro degradation of human tropoelastin by MMP-12 and the generation of matrikines from domain 24. Matrix Biol. 2009;28(2):84-91.

68. Palecek F, Palecekova M, Aviado DM. Emphysema in immature rats. Condition produced by tracheal constriction and papain. Arch Environ Health. 1967;15(3):332-342.

69. Kaplan PD, Kuhn C, Pierce JA. The induction of emphysema with elastase. I. The evolution of the lesion and the influence of serum. J Lab Clin Med. 1973;82(3):349-356.

70. Larsson C. Natural history and life expectancy in severe $\alpha 1$-antitrypsin deficiency, Pi Z. Acta Med Scand.1978;204(5):345-351.

71. Stone PJ. The elastase-antielastase hypothesis of the pathogenesis of emphysema. Clin Chest Med. 1983;4(3):405-412.

72. Woodruff PG, et al. A distinctive alveolar macrophage activation state induced by cigarette smoking. Am J Respir Crit Care Med. 2005;172(11):1383-1392.

73. Hautamaki RD, Kobayashi DK, Senior RM, Shapiro SD. Requirement for macrophage elastase for cigarette smoke-induced emphysema in mice. Science. 1997;277(5334):2002-2004.

74. Houghton AM, et al. Elastin fragments drive disease progression in a murine model of emphysema. J Clin Invest. 2006;116(3):753-759.

75. Hunninghake GM, et al. MMP12, lung function, and COPD in high-risk populations. $N$ Engl J Med. 2009;361(27):2599-2608.

76. Jiang D, Liang J, Noble PW. Hyaluronan in tissue injury and repair. Annu Rev Cell Dev Biol. 2007;23:435-461.

77. Sato H, et al. Antioxidant activity of synovial fluid, hyaluronic acid, and two subcomponents of hyaluronic acid. Synovial fluid scavenging effect is enhanced in rheumatoid arthritis patients. Arthritis Rheum. 1988;31(1):63-71.

78. Scheibner KA, Lutz MA, Boodoo S, Fenton MJ, Powell JD, Horton MR. Hyaluronan fragments act as an endogenous danger signal by engaging TLR2. J Immunol. 2006;177(2):1272-1281.

79. Todd JL, et al. Hyaluronan contributes to bronchiolitis obliterans syndrome and stimulates lung allograft rejection through activation of innate immunity. Am J Respir Crit Care Med. 2014;189(5):556-566.

80. Fieber C, et al. Hyaluronan-oligosaccharideinduced transcription of metalloproteases. J Cell Sci. 2004;117(pt 2):359-367.

81. Henke CA, Roongta U, Mickelson DJ, Knutson JR, McCarthy JB. CD44-related chondroitin sulfate proteoglycan, a cell surface receptor implicated with tumor cell invasion, mediates endothelial cell migration on fibrinogen and invasion into a fibrin matrix. JClin Invest. 1996;97(11):2541-2552.

82. Jiang D, Liang J, Noble PW. Hyaluronan as an immune regulator in human diseases. Physiol Rev. 2011;91(1):221-264.

83. Sherman L, Sleeman J, Herrlich P, Ponta H. Hyaluronate receptors: key players in growth, differentiation, migration and tumor progression. Curr 
Opin Cell Biol. 1994;6(5):726-733.

84. Teder P, et al. Resolution of lung inflammation by CD44. Science. 2002;296(5565):155-158.

85. Jiang D, et al. Regulation of lung injury and repair by Toll-like receptors and hyaluronan. Nat Med. 2005;11(11):1173-1179.

86. Rakoff-Nahoum S, Paglino J, Eslami-Varzaneh F, Edberg S, Medzhitov R. Recognition of commensal microflora by toll-like receptors is required for intestinal homeostasis. Cell. 2004;118(2):229-241.

87. Aumailley M, et al. A simplified laminin nomenclature. Matrix Biol. 2005;24(5):326-332.

88. Yurchenco PD. Basement membranes: cell scaffoldings and signaling platforms. Cold Spring Harb Perspect Biol. 2011;3(2):a004911.

89. Aumailley M. The laminin family. Cell Adh Migr. 2013;7(1):48-55.

90. Chou RH, Lin SC, Wen HC, Wu CW, Chang WS Epigenetic activation of human kallikrein 13 enhances malignancy of lung adenocarcinoma by promoting $\mathrm{N}$-cadherin expression and laminin degradation. Biochem Biophys Res Commun. 2011;409(3):442-447.

91. Roychaudhuri R, et al. ADAM9 is a novel product of polymorphonuclear neutrophils: regulation of expression and contributions to extracellular matrix protein degradation during acute lung injury. J Immunol. 2014;193(5):2469-2482.

92. Koster J, Borradori L, Sonnenberg A. Hemidesmosomes: molecular organization and their importance for cell adhesion and disease. Handb Exp Pharmacol. 2004;(165):243-280.

93. Schenk S, et al. Binding to EGF receptor of a laminin-5 EGF-like fragment liberated during MMP-dependent mammary gland involution. J Cell Biol. 2003;161(1):197-209.

94. Koshikawa N, et al. Proteolytic processing of laminin- 5 by MT1-MMP in tissues and its effects on epithelial cell morphology. FASEB J. 2004;18(2):364-366.

95. Sadowski T, et al. Matrix metalloproteinase
19 processes the laminin $5 \gamma 2$ chain and induces epithelial cell migration. Cell Mol Life Sci. 2005;62(7-8):870-880.

96. Pirilä E, et al. Matrix metalloproteinases process the laminin-5 $\gamma 2$-chain and regulate epithelial cell migration. Biochem Biophys Res Commun. 2003;303(4):1012-1017.

97. Ding BS, et al. Endothelial-derived angiocrine signals induce and sustain regenerative lung alveolarization. Cell. 2011;147(3):539-553.

98. Mydel P, et al. Neutrophil elastase cleaves laminin-332 (laminin-5) generating peptides that are chemotactic for neutrophils. J Biol Chem. 2008;283(15):9513-9522.

99. Lee J, Horuk R, Rice GC, Bennett GL, Camerato T, Wood WI. Characterization of two high affinity human interleukin-8 receptors. J Biol Chem. 1992;267(23):16283-16287.

100.Houghton AM. Matrix metalloproteinases in destructive lung disease. Matrix Biol. 2015; 44-46:167-174.

101.Akthar S, et al. Matrikines are key regulators in modulating the amplitude of lung inflammation in acute pulmonary infection. Nat Commun. 2015;6:8423.

102. University of Alabama at Birmingham. Trial of Doxycycline to Reduce Sputum MMP-9 Activity in Adult Cystic Fibrosis (CF) Patient (DOXY). ClinicalTrials.gov website. https:// clinicaltrials.gov/ct2/show/NCT01112059?ter$\mathrm{m}=$ NCT01112059\&rank=1. Updated January 26, 2016. Accessed July 20, 2016.

103. He J, Turino GM, Lin YY. Characterization of peptide fragments from lung elastin degradation in chronic obstructive pulmonary disease. Exp Lung Res. 2010;36(9):548-557.

104.Deslee G, et al. Elastin expression in very severe human COPD. Eur Respir J. 2009;34(2):324-331.

105. Robertoni FS, et al. Collagenase mRNA overexpression and decreased extracellular matrix components are early events in the pathogenesis of emphysema. PLoS One. 2015;10(6):e0129590.

106.Jesudason R, et al. Mechanical forces regulate elastase activity and binding site availability in lung elastin. Biophys J. 2010;99(9):3076-3083.

107. Meiners S, Eickelberg O, Königshoff M. Hallmarks of the ageing lung. Eur Respir J. 2015;45(3):807-827.

108. Jenkins RG, et al. Longitudinal change in collagen degradation biomarkers in idiopathic pulmonary fibrosis: an analysis from the prospective, multicentre PROFILE study. Lancet Respir Med. 2015;3(6):462-472.

109. Churg A, Wang R, Wang X, Onnervik PO, Thim K, Wright JL. Effect of an MMP-9/MMP-12 inhibitor on smoke-induced emphysema and airway remodelling in guinea pigs. Thorax. 2007;62(8):706-713.

110. Rowe SM, et al. Potential role of high-mobility group box 1 in cystic fibrosis airway disease. $A m \mathrm{~J}$ Respir Crit Care Med. 2008;178(8):822-831.

111. Calışkan B, Banoglu E. Overview of recent drug discovery approaches for new generation leukotriene A4 hydrolase inhibitors. Expert Opin Drug Discov. 2013;8(1):49-63.

112. Lin L, et al. Neutrophil elastase cleaves the murine hemidesmosomal protein BP180/type XVII collagen and generates degradation products that modulate experimental bullous pemphigoid. Matrix Biol. 2012;31(1):38-44.

113. Fisher GJ, et al. Collagen fragmentation promotes oxidative stress and elevates matrix metalloproteinase-1 in fibroblasts in aged human skin. Am J Pathol. 2009;174(1):101-114.

114. Davis GE, Bayless KJ, Davis MJ, Meininger GA. Regulation of tissue injury responses by the exposure of matricryptic sites within extracellular matrix molecules. Am J Pathol. 2000;156(5):1489-1498.

115. Adair-Kirk TL, Senior RM. Fragments of extracellular matrix as mediators of inflammation. Int $J$ Biochem Cell Biol. 2008;40(6-7):1101-1110. 